\title{
The plasmonic coupling of metal nanoparticles and its implication for scanning near-field optical microscope characterization
}

Researchers at State Key Laboratory for Mesoscopic Physics and Department of Physics, Peking University (PKU), have recently shown that an otherwise forbidden longitudinal surface plasmon mode of a gold nanorod can be excited by normally incident linearly-polarized light if it is coupled with a nanoparticle with proper plasmonic resonant frequency, volume, and composition ${ }^{[1]}$.

This induced excitation of the forbidden mode results from the broken symmetry of the local electric field experienced by the nanorod caused by the charge pileup on the guest nanoparticle in the exciting field, according to their study.

Surface plasmons are collective oscillations of the free electrons coupled with the electromagnetic field. Nanoparticles of noble metals can sustain localized surface plasmon resonances at or close to the visible frequency. Due to their novel optical properties such as mode confinement and field enhancement, and the consequent promise for a wide range of applications, plasmonic nanostructure is currently a hot topic of research. Understanding the coupling of two or more plasmonic resonant nanoparticles is not only of fundamental importance in studying plasmons themselves, but also is cru- cial for designing future plasmonic devices.

Scientists have investigated this issue in a number of ways. Jain et al. noticed in their experiments that a dimer of gold nanorods assembled in a side-by-side fashion has a longitudinal surface plasmon resonance blue-shifted from that of an isolated gold nanorod, but has a transverse resonance red-shifted from that of an isolated nanorod. The closer the two nanorods get, the larger the shift. The shift of the longitudinal resonance increases with increasing nanorod's aspect ratio for both side-by-side and end-to-end assemblies. If two nanorods with different aspect ratio is coupled, the dimer will have peaks around the resonances of the constituent nanorods when isolated. They found these to be consistent with the molecular exciton coupling theory ${ }^{[2]}$. Su et al. ${ }^{[3]}$ found from simulations and experiments that the resonance shift of a dimer of elliptical metal particles decays approximately exponentially with increasing particle separation and becomes negligible "when the gap between the two particles exceeds about 2.5 times the particle short-axis length". Jain et al. ${ }^{[4]}$ further discovered that this exponential decay depends on the particle separation scaled by the particle size. Prodan et al. ${ }^{[5]}$ provided a good theoretical picture of plasmonic coupling with their plasmon hybridization model.

However, all the above works treated the coupled nanoparticles as a whole, so they gave little insight into how one nanoparticle affects the plasmonic properties of the other through coupling. The PKU group adopted a new approach, treating one nanoparticle as the host and the other as the guest. Their focus was how the guest nanoparticle modifies the host nanoparticle's plasmonic resonances.

Their approach is particularly suitable for understanding the near-field optical characterization of metal nanostructures. As a unique optical characterization tool in the field of nano-optics that can achieve subwavelength optical resolutions, the scanning near-field optical microscope employs a probe scanning in the vicinity of the nano-sized metal sample which often complicates the interpretation of the obtained signal. The results here demonstrate using all-dielectric probes will preserve the intrinsic plasmonic properties of the sample while metal-coated or metal probes will generally modify these properties.

LI Zhi \& GONG QiHuang State Key Laboratory for Mesoscopic and Department of Physics, Peking University, Beijing 100871, China

1 Yao H M, Li Z, Gong Q H. Coupling-induced excitation of a forbidden surface plasmon mode of a gold nanorod. Sci China Ser G-Phys Mech Astron, 2009, 52: $1129-1138$

2 Jain P K, Eustis S, El-Sayed M A. Plasmon coupling in nanorod assemblies: Optical absorption, discrete dipole approximation simulation, and exciton-coupling model. J Phys Chem B, 2006, 110: 18243-18253

3 Su K H, Wei Q H, Zhang X, et al. Interparticle coupling effects on plasmon resonances of nanogold particles. Nano Lett, 2003, 3: 1087-1090

4 Jain P K, Huang W, El-Sayed M A. On the universal scaling behavior of the distance decay of plasmon coupling in metal nanoparticle pairs: A plasmon ruler equation. Nano Lett, 2007, 7: 2080-2088

5 Prodan E, Radloff C, Halas N J, et al. A hybridization model for the plasmon response of complex nanostructures. Science, 2003, 302(5644): 419422

doi: 10.1007/s11434-009-0638-9 